\title{
Propiedades, modificaciones Y usos de los almidones, CON MIRAS A LA OBTENCIÓN DE PELÍCULAS BIODEGRADABLES. ARTÍCULO DE REVISIÓN
}

\author{
Properties, MOdifications, AND USES OF STARCHES OBTAINING \\ BIODEGRADABLE FILIMS. REVIEW ARTICLE
}

Claudio Raúl Bernal Bustos*

Juan Pablo Guarnizo Sánchez ${ }^{* *}$

Andrés Guevara***

Daniela Suarez ${ }^{* * * * *}$

Nicolás Lozano******

Simón Castillo ${ }^{* * * * * * *}$

Recibido: 14 de julio de 2017

Aceptado: 9 de octubre de 2017

\section{Resumen}

En este artículo se analizan las propiedades, características y condiciones para obtener estructuras poliméricas (películas) utilizando almidón de diversas fuentes naturales, entre ellas: maíz, arroz, papa, yuca, quinua, banano. El propósito de esta revisión, por una parte, es precisar tres variables que están íntimamente relacionadas en la obtención de dichas estructuras con potencial biodegradable: contenido de amilosa, diámetro de partícula y temperatura de gelatinización, y por otra, definir las cantidades de los ingredientes para la formulación de la película, tales como el porcentaje de almidón y el plastificante, así como las condiciones de secado. El trabajo expone el efecto de cada propiedad en las características mecánicas y ópticas de la estructura, así como selecciona almidones favorables para esta aplicación. Para llegar a estos resultados, se exponen las generalidades de los almidones (del almidón de quinua, particularmente), sus propiedades fisicoquímicas y sus posibles modificaciones. Por último, se desarrolla el tema con base en la información presentada.

Palabras clave: almidón, quinua, modificación física y química, propiedades fisicoquímicas, estructuras poliméricas biodegradables.

\footnotetext{
* Docente investigador, estudiante Doctorado (ED) Avances en Ciencias y Biotecnología Alimentaria, director del Centro de Bioprospección e Ingeniería Química Aplicada al Estudio de Biomoléculas e Industria (BIQA), Fundación Universidad de América, Bogotá, Colombia. ORCID:http://orcid.org/0000-0002-0750-0142. claudio.bernal@ investigadores.uamerica.edu.co

** Estudiante Programa de Ingeniería Química, coinvestigador Centro BIQA-Estructuras Híbridas Poliméricas (EHP), Fundación Universidad de América, Bogotá, Colombia. juan.guarnizo@estudiantes.uamerica.edu.co

${ }^{* * *}$ Estudiante Programa de Ingeniería Química, semillero Centro BIQA-EHP, Fundación Universidad de América, Bogotá, Colombia. andres.guevara3@estudiantes.uamerica.edu.co

${ }^{\star \star \star \star \star}$ Estudiante Programa de Ingeniería Química, Semillero Centro BIQA-EHP, Fundación Universidad de América, Bogotá, Colombia. daniela.suarez3@estudiantes.uamerica.edu.co

**** Estudiante Programa de Ingeniería Química, Semillero Centro BIQA-EHP, Fundación Universidad de América, Bogotá, Colombia. nicolas.lozano2@estudiantes.uamerica.edu.co

${ }_{* * * * *}$ Estudiante Programa de Ingeniería Química, Semillero Centro BIQA-EHP, Fundación Universidad de América, Bogotá, Colombia. nicolas.lozano2@estudiantes.uamerica.edu.co

${ }_{* \star * \star \star \star \star}$ Estudiante Programa de Ingeniería Química, Semillero Centro BIQA-EHP, Fundación Universidad de América, Bogotá, Colombia. simon.castillo@ estudiantes.uamerica.edu.co
} 


\section{Abstract}

This article analyzes the properties, characteristics and conditions for obtaining polymeric structures; films using starch extracted from various natural sources, such as corn, rice, potatoe, cassava, quinoa, banana. The purpose of this review, on the one hand, is to specify three variables that are closely related to obtaining such structures with biodegradable potential such as: amylose content, particle diameter and gelatinization temperature; and on the other hand, to define the amounts of ingredients for the formulation of starch percentage, plasticizer and drying conditions. The work exposes the effect of each property on the mechanical and optical characteristics of the structure, as well as selecting favorable starches for this application. To achieve these results, the generalities of the starches (of the quinoa starch, in particular), their physicochemical properties and their possible modifications are shown. Finally, the topic is developed based on the information presented.

Keywords: starch, quinoa, physical and chemical modification, physicochemical properties, biodegradable polymeric structures.

\section{INTRODUCCIÓN}

La Fundación Universidad de América, a través del Centro de Bioprospección e Ingeniería Química Aplicada al Estudio de Biomoléculas e Industria (CBIQA), está desarrollando un programa de investigación de la quinua en el periodo 2010-2020. En la primera fase de este proyecto (20102015) se realizaron ensayos experimentales alrededor de modificaciones físicas, químicas y biotecnológicas del almidón de la especie Chenopodium quinua Willd. En la segunda fase (2015-2020) se tiene proyectado que el CBIQA genere evidencia científica para que el Gobierno, los productores y los transformadores consoliden la cadena productiva y de valor de la quinua bajo un nuevo enfoque de negocio e investigación (Bernal, Villegas, Sandoval, Lache y Correa, 2015). El enfoque científico enfatiza, por una parte, en la estructura y morfología del grano-semilla de quinua con la perspectiva de obtener unidades primarias de partida (UPP); no obstante las UPP obtenidas mediante procesos físicos, la investigación se centra en el almidón contenido en el perisperma del grano-semilla. Por otra parte y respecto al enfoque de negocio, el programa enfatiza en la definición de un "patrón de uso" del almidón, en el que se evidencia su gran potencial para la industria cosmética y alimentaria, así como sus diversas aplicaciones (aditivo industrial, por ejemplo).

Con el propósito de avanzar en el enfoque científico del almidón, en esta revisión se estudian los polímeros con potencial para la elaboración de películas según el análisis de las propiedades más necesarias para este fin: contenido de amilosa, diámetro de partícula, hinchamiento de los granos, temperatura de gelatinización, pegado y retrodegración; propiedades que están directamente relacionadas con tres aspectos: propiedades de la película, posible aplicación y potencial biodegradable de los polímeros amiláceos. Sin embargo, establecidas las características del almidón, esta revisión se orienta a las propiedades de la película.

La producción de plásticos depende de la disponibilidad de recursos petroleros; estos recursos muestran actualmente una mayor limitación en su uso por el impacto que generan sobre el ambiente (Basiak, Lenart y Debeaufort, 2017). Específicamente, los rellenos sanitarios son unos de los más afectados; a nivel mundial, los plásticos ocupan el $10 \%$ de su capacidad (Hoornweg y Bhada, 2012). El gran volumen acumulado de estos residuos y su largo periodo de biodegradabilidad exigen explorar la posibilidad de usar otros productos más amigables con el entorno.

Esta situación ha llamado la atención de la comunidad internacional; particularmente, el Servicio de Información Comunitario sobre Investigación y Desarrollo (CORDIS), quienes vienen 
incentivando el uso de los "polímeros verdes" con el propósito de reemplazar o disminuir los polímeros de origen petroquímico en un gran número de aplicaciones (ver, por ejemplo, Comisión Europera, 2015). Bajo este precepto, los polímeros naturales son parte de las alternativas a considerar, ya que se pueden obtener de diferentes fuentes y a bajo costo.

Los polímeros naturales, como proteínas, celulosa, almidón, glicógeno, sedas (poliamidas), lana (proteínas), caucho (Gonzalez-Gutierrez, Partal, Garcia-Morales y Gallegos, 2010) son sustancias candidatas. Como materias primas, estos polímeros podrían mitigar la relación entre productosambiente; relación que debe ir en función de causar el mínimo impacto sobre el medioambiente. Este trabajo revisa la utilización del almidón en la manufactura de películas con potencial biodegradable (Averous, Fauconnier, Moro y Fringant, 2000; Mathew y Dufresne, 2002), centrando la atención en el uso del almidón de la especie Chenopodium quinua Willd. en dicha manufactura.

\section{Almidón}

El almidón, reserva energética de las plantas, es almacenado de forma granular y se puede encontrar de manera abundante en semillas, raíces, tubérculos, tallos y hojas (Jane, 2009; Jiménez et ál., 2012). Este polímero está compuesto principalmente por amilosa y amilopectina, en una relación 18-33\% y 72-78 \%, respectivamente (Buléon, Colonna, Planchot y Ball, 1998; Masina et ál., 2017). La amilosa y la amilopectina presentan propiedades especificas (ver tabla 1) que afectan las propiedades del almidón y varían dependiendo de su origen (Lourdin, Valle y Colonna, 1995; Robyt, 2009).

Generalmente, las diversas clases de almidones permiten obtener estructuras-películas con potencial biodegradable, que difieren en características como el espesor-grosor, solubilidad en agua y ácidos, resistencia a la tracción, porcentaje de elongación, entre otras (Fakhouri, Martelli, Caon, Velasco y Mei, 2015).

A partir del almidón como materia prima, existe la posibilidad de obtener dos clases de estructuras poliméricas: un polímero termoplástico que se produce a partir de un proceso en seco, y una película (film) que se obtiene a partir de un proceso en medio húmedo. La principal diferencia entre los dos procesos es la cantidad de agua con la que se realiza, debido a que el método húmedo utiliza agua como plastificante en exceso (Jiménez, Fabra, Talens y Chiralt, 2012). Sin embargo, se debe considerar el uso de aditivos en el desarrollo de una u otra estructura polimérica, pues inciden tanto en las características del producto final, como en su proceso de obtención. Entre los aditivos más usados están los plastificantes, como glicerina, sorbitol, xilitol o alcohol polivinílico (Pacheco, Flores y Romina, 2014), que aportan humectación al polímero. En algunos casos, la obtención del producto requiere el uso de lubricantes como aceites naturales, puesto que brindan manejabilidad y no permiten la adherencia en las superficies de trabajo (Meneses, Corrales y Valencia, 2007).

Las propiedades fisicoquímicas tienen una relación directa con las propiedades y características de la estructura, por lo que se hace necesario identificar y analizar las propiedades de cada almidón. En la tabla 1 se elabora una matriz comparativa entre diversas variedades de almidón y las propiedades fisicoquímicas, que varían dependiendo de su origen y genética (Jane, 2009; Lourdin et ál., 1995). 


\begin{tabular}{|c|c|c|c|c|c|c|c|c|c|c|c|c|c|c|}
\hline \multirow{8}{*}{ 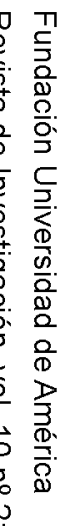 } & \multicolumn{14}{|c|}{ Tabla 1. Datos comparativos de las principales propiedades fisicoquímicas, según la fuente de almidón } \\
\hline & $\begin{array}{l}\text { Fuente de } \\
\text { almidón }\end{array}$ & $\begin{array}{c}\text { Diametro } \\
\text { particula ( }(\mu \mathrm{m})\end{array}$ & Amilosa \% & $\begin{array}{l}\text { Humedad } \\
\%\end{array}$ & $\begin{array}{l}\text { Temp Onset } \\
{ }^{\circ} \mathrm{C}\end{array}$ & $\begin{array}{l}\text { Temp } \\
\text { Peak }{ }^{\circ} \mathrm{C}\end{array}$ & $\begin{array}{l}\text { Temp } \\
\text { Finall }\end{array}$ & $\begin{array}{c}\text { Índice De } \\
\text { Solubilidad En } \\
\text { Agua \% }\end{array}$ & $\begin{array}{c}\text { Poder de } \\
\text { Hinchamiento } \\
(g / g)\end{array}$ & $\begin{array}{c}\text { Cambio Entalpia } \\
\text { Gelatinización }(J / g)\end{array}$ & $\begin{array}{l}\text { Dureza } \\
\text { (g) }\end{array}$ & $\begin{array}{l}\text { Adhesion } \\
\text { (g. s) }\end{array}$ & Cohesión & Cristalinidad \% \\
\hline & Normal potato a & $20-110$ & $20.1-31$ & - & $59.72-66.2$ & $62.9-69.6$ & $67.28-75.4$ & 82 & 1159 & $12.55-17.9$ & - & - & . & . \\
\hline & Solanum tuberosum (potato) b & $15-110$ & 19.1 & $\cdot$ & $\cdot$ & $\cdot$ & - & 82 & 1159 & - & - & - & - & - \\
\hline & $\begin{array}{l}\text { Normal } \\
\text { corn a }\end{array}$ & $\begin{array}{l}\text { 1-7 pequeńos, } \\
15-20 \text { largos. }\end{array}$ & $22.4-32.5$ & - & 62.3 & 67.7 & 84.3 & 22 & 22 & 14 & - & - & $\cdot$ & - \\
\hline & Waxy corn a & $\begin{array}{l}\text { 1-7 pequeños, } \\
15-20 \text { largos. }\end{array}$ & $1.4-2.7$ & - & 66.6 & 73.6 & - & - & - & 14.2 & - & - & - & - \\
\hline & High amylose corn ${ }^{2}$ & $\begin{array}{l}\text { 1-7 pequeños, } \\
15-20 \text { largos. }\end{array}$ & $42.6-67.8$ & - & 60.8 & 73.7 & - & 12.4 & 6.3 & 13.7 & - & - & - & - \\
\hline & Normal rice a & 3.5 & $5-28.4$ & - & 62 & 67.4 & 97.5 & $11-18$ & $23-30$ & 11 & - & - & - & - \\
\hline & Waxy rice = & $3-5$ & $0-2$ & - & $66.1-74.9$ & $70.4-78.8$ & - & $2.3-3.2$ & $45-50$ & $77-12.1$ & - & . & . & . \\
\hline & High amylose rice a & 3-5 & $25-33$ & - & & - & - & & & - & - & - & - & \\
\hline & Normal wheat a & - & $18-30$ & $\cdot$ & 57.1 & 61.6 & 66.2 & 1.55 & $18.3-26.6$ & 10.7 & - & - & - & . \\
\hline & Dicscorea cayanesis (batata) ${ }^{b}$ & $6-100$ & $22.8-30$ & - & 68 & - & 74.5 & 13.8 & 16.9 & - & - & - & - & - \\
\hline & Discorea dumetorum (Batata) & $28.5-30.6$ & $21,6-27$ & - & 65.5 & - & 72.5 & 16.8 & 18.6 & - & - & - & - & - \\
\hline & Discorea rotundata (Batata) ${ }^{\mathrm{b}}$ & $10-22.7$ & 22,4 & - & 63.5 & - & 71 & 11.9 & 21.5 & - & - & - & - & \\
\hline & $\begin{array}{l}\text { Colocassia esculenta (old } \\
\text { coco yam })^{\mathrm{b}}\end{array}$ & $3.0-3.5$ & 21,4 & - & 43 & - & 63.3 & - & - & 6.8 & - & - & - & 45 \\
\hline & Banana (Gros Michel)' ' & $\begin{array}{c}\text { 10-20 } \\
\text { longitu35-70 }\end{array}$ & 22.76 & 4.36 & - & - & - & - & - & - & - & - & - & - \\
\hline & Banana (Dominico Harton) ' & $\begin{array}{l}20-30 \text { y } 50-65 \text { de } \\
\text { largo. }\end{array}$ & 31.12 & 8.01 & - & - & - & - & - & - & - & - & - & - \\
\hline & Banana (FHIA 20) ' & $\begin{array}{l}\text { 20-30 y } 50-65 \text { de } \\
\text { largo. }\end{array}$ & 28.58 & 7.92 & - & - & - & - & - & - & - & - & - & - \\
\hline & Plátano amarillo ${ }^{\circ}$ & $11.22-41$ & 24.36 & 8.62 & 64.99 & 68.68 & 73.9 & - & - & 8.59 & - & - & - & - \\
\hline & Plátano blanco ${ }^{\circ}$ & $10-33$ & 24.24 & 8.44 & 68.08 & 71.88 & 77.15 & - & - & 15.02 & - & - & - & - \\
\hline & Plátano (Tanduk) ${ }^{1}$ & 23.91 & 26.084 & 8.829 & 63.13 & 83.83 & 122.67 & & & - & 85.83 & - & 0.44 & \\
\hline & Plátano (Nangka) ' & 38.97 & 31.789 & 9.837 & 65.19 & 84.25 & 123.8 & - & - & - & 63.13 & & 0.35 & - \\
\hline & Name (bolañero) ${ }^{d}$ & 10-100 & 27.89 & 9.75 & - & 78 & - & - & - & - & - & - & - & - \\
\hline & Ñame (Diamante22) ${ }^{\circ}$ & $10-100$ & 27.55 & 10.09 & - & 79 & - & - & - & - & - & - & - & - \\
\hline & Ñame (ecuatoriano) ${ }^{d}$ & 10-100 & 25.87 & 8.66 & - & 77 & - & - & - & - & - & - & - & - \\
\hline & Name (pico botella) ${ }^{d}$ & $10-100$ & 25.95 & 10.02 & . & 75 & - & - & - & - & - & - & - & - \\
\hline
\end{tabular}




\begin{tabular}{|c|c|c|c|c|c|c|c|c|c|c|c|c|c|}
\hline $\begin{array}{l}\text { Fuente de } \\
\text { almidón }\end{array}$ & $\begin{array}{c}\text { Diámetro } \\
\text { particula }(\boldsymbol{\mu m})\end{array}$ & Amilosa \% & $\begin{array}{l}\text { Humedad } \\
\%\end{array}$ & $\begin{array}{l}\text { Temp Onset } \\
{ }^{\circ} \mathrm{C} C\end{array}$ & $\begin{array}{l}\text { Temp } \\
\text { Peak }{ }^{\circ} \mathrm{C}\end{array}$ & $\begin{array}{l}\text { Temp } \\
\text { Final }{ }^{\circ} \mathrm{C}\end{array}$ & $\begin{array}{c}\text { Índice De } \\
\text { Solubilidad En } \\
\text { Agua \% }\end{array}$ & $\begin{array}{c}\text { Poder de } \\
\text { Hinchamiento } \\
(\mathrm{g} / \mathrm{g})\end{array}$ & $\begin{array}{c}\text { Cambio Entalpia } \\
\text { Gelatinizaciôn }(\mathrm{J} / \mathrm{g})\end{array}$ & $\begin{array}{l}\text { Dureza } \\
\text { (g) }\end{array}$ & $\begin{array}{l}\text { Adhesión } \\
\text { (g. s) }\end{array}$ & Cohesión & Cristalinidad \% \\
\hline Yuca (ICA-C523-7) d & $10-100$ & 14.67 & 8.47 & - & 61 & - & - & - & - & - & - & - & - \\
\hline Yuca $(\mathrm{HCM}-1)^{d}$ & 10-100 & 14.23 & 7.8 & - & 62 & - & - & - & - & - & - & - & - \\
\hline Yuca (MBra 383) d & $10-100$ & 14.23 & 7.8 & - & 63 & - & - & . & . & - & . & . & . \\
\hline Papa (ICA Nariño) व & $10-100$ & 24 & 8.5 & . & 66 & - & - & . & . & - & . & . & . \\
\hline Quinoa $\mathrm{g}^{\mathrm{h}}$ & $1-3$ & $10-21.2$ & 10.1 & 52.3 & 59.2 & 69.5 & 48.6 & 23.2 & 13.6 & 20.8 & 158 & 0.63 & 35.4 \\
\hline Amaranto n & - & 7.8 & - & $66,$. & 74.5 & 86.9 & - & - & 2.58 & - & - & - & 45.5 \\
\hline Curcuma angusiffolia * & $6.3-31.7$ & 31.4 & . & . & 78.2 & - & 40 & . & . & - & . & . & . \\
\hline
\end{tabular}

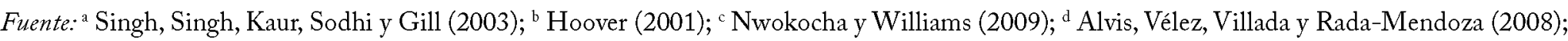

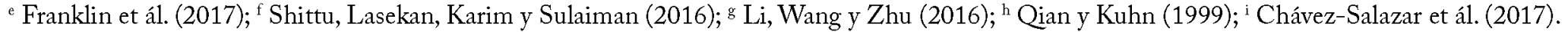


Investigaciones realizadas a nivel mundial con diferentes almidones muestran que cada uno tiene un comportamiento particular. Por ejemplo, la elaboración de biopolímeros con base en el almidón de trigo (Triticum durum) es uno de los procesos más utilizados debido a que este almidón no es tóxico para el ser humano (Patni, Yadava, Agarwal y Maroo, 2014). La estructura obtenida del gluten de trigo es degradada por el ambiente en un tiempo aproximado de 36 días, lo que lo hace un material ideal, comparándolo con los plásticos producidos hoy en día; además, estos materiales pueden tener características muy similares a las de sustancias como el polipropileno y los epóxidos (Patni et ál., 2014). También se ha evaluado la incidencia del aserrín en las propiedades del biopolímero (Bootklad, Chantarak y Kaewtatip, 2016), pues conlleva un amento en sus características de fuerza y resistencia al agua, aunque incrementa el tiempo de biodegradabilidad (Bootklad et ál., 2016).

El almidón de plátano (Musa x paradisiaca L.) también ha sido objeto de estudio. La investigación de Pelissari, Andrade-Mahecha, Amaral y Menegalli (2013) obtuvo una película con resistencia mecánica y poca flexibilidad, liviana y baja opacidad, baja solubilidad en agua y una microestructura compacta; particularidades que hacen que este sea un material prometedor para la formulación de recubrimientos. Para este caso, se utilizó almidón modificado (oxidado) de plátano Musa paradisiaca L. y alcohol polivinílico para preparar la película, lo que ocasiona que algunas propiedades mecánicas (modulo elástico, tensión de rotura o alargamiento a la rotura) mejoren, mientras que otras propiedades (permeabilidad al vapor de agua) disminuyan (Palma-Rodríguez et ál., 2012). También fueron agregadas otras sustancias para evaluar el efecto que tenían sobre las propiedades de la película; el aceite de canela, por ejemplo, origina una disminución en la permeabilidad al vapor de agua y la solubilidad, además de producir un efecto antibacteriano que no altera las propiedades mecánicas de la película (Romero-Bastida, Zamudio-Flores y Bello-Pérez, 2011); asimismo, la adición de caucho natural genera una degradabilidad inversamente proporcional a la humedad y una cristalinidad directamente proporcional a la humedad (Ramírez-Hernández, Aparicio-Saguilán, Reynoso-Meza y Carrillo-Ahumada, 2017).

De manera similar se ha usado el almidón de papa (Solanum tuberosum). En este caso se encontró que el principal plastificante y compatibilizante es la glicerina (Podshivalov, Zakharova, Glazacheva y Uspenskaya, 2017). Esto se da debido a que el comportamiento presentado tiene más resistencia a la ruptura que cualquier otro material. Si se adiciona otro tipo de aditivos, como catequina o NCA (Nanocristales de Almidón) (Sessini, Arrieta, Kenny y Peponi, 2016), aumentará mucho más esta característica. Con respecto a sus propiedades fisicoquímicas, se encontró que los gránulos de almidón se ven afectados debido a que en la microscopia óptica (durante la filtración la amilasa intragranular) los gránulos empiezan a romperse, por lo que los enlaces de hidrogeno se separan, se solubiliza la amilosa y la amilopectina y disminuye la temperatura de la solución (Martins et ál., 2016). En cuanto a las propiedades mecánicas (como la resistencia y la elasticidad), la transparencia y el espesor de la película dependen fuertemente del contenido de almidón y su porción en peso (Podshivalov et ál., 2017). Este cambio de las propiedades del almidón durante la gelatinización implica una hinchazón del grano en todos los casos (Tan, Torley y Halley, 2008). La gelatinización supone altas concentraciones de almidón, que conducen a la formación de la morfología de los materiales con gránulos coalescidos frenados de fase bicontinua de almidón en gránulos más pequeños, es decir, dos fases, lo que resulta en una diferencia de las propiedades viscoelásticas de los componentes en el proceso de gelificación de la solución de la película (Tan et ál., 2008).

Como se mencionó anteriormente, la variedad de estructuras obtenidas se debe a la diferencia entre las propiedades fisicoquímicas de los almidones, ya que cada una confiere una característica distinta al biopolímero. Las propiedades que se deben considerar se escogen en función del uso que se le quiera brindar, es por esto que la matriz comparativa de la tabla 1 resulta de gran utilidad para confrontar y seleccionar los almidones más idóneos según la aplicación y como posibles sustitutos 
de los plásticos actuales. La tabla 2 expone diversas formulaciones de películas que se han realizado a partir de almidones, considerando los agentes plastificantes, las condiciones de secado y otros posibles aditivos involucrados en el proceso. Actualmente, los avances realizados en este tema no solo se centran en las propiedades de los almidones, sino también en la funcionalización del almidón e, inclusive, en la hibridación de polímeros a partir de dos almidones, insertando nanocristales de uno de ellos. Esto con el fin de mejorar las propiedades de termo resistencia y flexibilidad, para que el plástico tenga una mayor vida útil respecto a otros plásticos y no genere un impacto medioambiental.

Una aplicación puntual para las estructuras elaboradas con almidón son las películas; estas se pueden utilizar en diversas aplicaciones, especialmente para recubrimientos o envolturas de alimentos. En este sentido, las películas deben ser flexibles, transparentes y tener una moderada resistencia a la tracción; este comportamiento va ligado a tres propiedades fisicoquímicas propias del almidón: diámetro de partícula, contenido de amilosa y temperatura de gelatinización.

Tabla 2. Formulaciones de películas utilizando como materia prima el almidón proveniente de diversas fuentes naturales

\begin{tabular}{|c|c|c|c|c|}
\hline Fuente de almidón & $\begin{array}{c}\text { Componentes } \\
\text { adicionales }\end{array}$ & Plastificantes & $\begin{array}{c}\text { Condiciones } \\
\text { gelatinización }\end{array}$ & Condiciones secado \\
\hline $\begin{array}{l}\text { Sagu (Maranta } \\
\text { arundinacea) }\end{array}$ & & Glicerina - Sorbitol & $85^{\circ} \mathrm{C}, 30 \mathrm{~min}$ & $40^{\circ} \mathrm{C}, 20 \mathrm{~h}$ \\
\hline $\begin{array}{l}\text { Quinoa (Chenopodium } \\
\text { quinua Willd) }\end{array}$ & & Glicerina & $97^{\circ} \mathrm{C}, 30 \mathrm{~min}$ & $34-50^{\circ} \mathrm{C}$ \\
\hline $\begin{array}{l}\text { Yuca (Manihot } \\
\text { esculenta) }\end{array}$ & & Glicerina & $70^{\circ} \mathrm{C}, 1 \mathrm{~min}$ & $30{ }^{\circ} \mathrm{C}, 18-24 \mathrm{~h}$ \\
\hline $\begin{array}{l}\text { Patata (Solanum } \\
\text { tuberosum) }\end{array}$ & & Glicerina & $70^{\circ} \mathrm{C}, 30 \mathrm{~min}$ & $60^{\circ} \mathrm{C}$ \\
\hline $\begin{array}{l}\text { Banana (Musa } \\
\text { acuminata) }\end{array}$ & & & $98^{\circ} \mathrm{C}, 30 \mathrm{~min}$ & $\begin{array}{l}4{ }^{\circ} \mathrm{C} \text { durante } 10 \text { días y } \\
\text { después } 40^{\circ} \mathrm{C} \text { por } 48 \mathrm{~h}\end{array}$ \\
\hline $\begin{array}{l}\text { Yuca (Manihot } \\
\text { esculenta) }\end{array}$ & Chitosan & Glicerina & $125^{\circ} \mathrm{C}, 30 \mathrm{~min}$ & $25^{\circ} \mathrm{C}, 48 \mathrm{~h}$ \\
\hline $\begin{array}{l}\text { Native Patata (Solanum } \\
\text { tuberosum) }\end{array}$ & & Glicerina - Sorbitol & $90^{\circ} \mathrm{C}, 5 \mathrm{~min}$ & $35^{\circ} \mathrm{C}$ Por al menos $4 \mathrm{~h}$ \\
\hline Arveja (Pisum sativum) & Beeswax & Glicerina & $\begin{array}{l}\text { Temperatura de } \\
\text { ebullición, } 15 \mathrm{~min}\end{array}$ & $\begin{array}{l}\text { Temperatura ambiente } \\
\text { por al menos } 40 \mathrm{~h}\end{array}$ \\
\hline Maiz (z.mayz) & $\begin{array}{l}\text { Aceite de } \\
\text { Girasol }\end{array}$ & Glicerina - Sorbitol & Medio alcalino & $60{ }^{\circ} \mathrm{C}, 8 \mathrm{~h}$ \\
\hline $\begin{array}{l}\text { Mango (Mangifera } \\
\text { indica), Banana (Musa } \\
\text { acuminata) }\end{array}$ & & Glicerina & $\begin{array}{l}\text { Gelatinización } \\
\text { en frio }\end{array}$ & $65^{\circ} \mathrm{C}, 5 \mathrm{~h}$ \\
\hline
\end{tabular}

Fuente: Jiménez, Fabra, Talens y Chiralt (2012)

\section{Metodología}

Este artículo evalúa el efecto que tiene las propiedades fisicoquímicas del almidón respecto al comportamiento mecánico y óptico de las estructuras poliméricas tipo película. Para llegar a este resultado se aborda como primera instancia el almidón, considerando las principales generalidades, características y propiedades que tiene; además, se trata de las principales modificaciones que el almidón puede sufrir, enfatizando en el almidón de quinua, ya que este resulta ser una materia prima que se puede utilizar en la elaboración de películas poliméricas. 
La revisión permitió construir relaciones directas e indirectas entre las propiedades del almidón y el comportamiento de la película; puntualmente, se identificó que un contenido elevado de amilosa mejoraba el comportamiento mecánico de la estructura, pero afectaba la transparencia del material haciendo que este se volviera más opaco. Igualmente, se determinó que un diámetro de partícula pequeño permitía una mayor dispersión del almidón en la solución de la película. Asimismo, se consideró la posibilidad de que las películas obtenidas a partir del almidón de quinua sean biodegradables, debido a que este es un polímero de origen natural, renovable y biodegradable (si es sometido a algunas situaciones particulares que lo lleven a su degradación, por ejemplo, la activación enzimática, entre otras), así como por su alta disponibilidad y bajo costo (Stagner, Alves y Narayan, 2012).

Finalmente, se plantea que los productos plastificados (películas) son una de las posibles estructuras que se pueden obtener a partir del almidón; entre estas, los almidones termoplásticos presentan mayor estabilidad frente aceites y grasas y pueden ser degradados completamente en 5 días en sistemas acuosos y en 45 días en ambientes controlados. También, es posible obtener otros productos como las espumas de almidón, que es un material antiestático, aislante y amortiguador y completamente biodegradable (Rubio y Guerrero, 2012).

\section{Marco teórico}

\section{Almidón}

Como se citó anteriormente, el almidón está compuesto por la amilosa y la amilopectina. La amilosa es un polímero lineal compuesto de glucosa que está conectado por enlaces a1-4 glucosidicos, aunque algunas unidades de glucosa están conectadas por enlaces a 1-6 (Buléon et ál., 1998); su peso molecular es de $10^{5}$ a $10^{6} \mathrm{~g} / \mathrm{mol}$ (Penaranda, Perilla y Algecira, 2008). La amilopectina, segundo componente mayoritario del almidón, es una molécula ramificada (Hernández-Medina, Torruco-Uco, Chel-Guerrero y Betancur-Ancona, 2008; Masina et ál., 2017) que presenta cientos de cadenas cortas a 1-4 glucano interrelacionadas por enlaces a 1-6 (Buléon et ál., 1998). La figura 1 representa los enlaces posibles de la glucosa en la amilosa y la amilopectina dentro de la molécula de almidón. El peso molecular de este polímero varía entre $10^{7}$ a $10^{8} \mathrm{~g} / \mathrm{mol}$ (Penaranda et ál., 2008). En la tabla 3 se presentan las principales propiedades de la amilosa y la amilopectina.
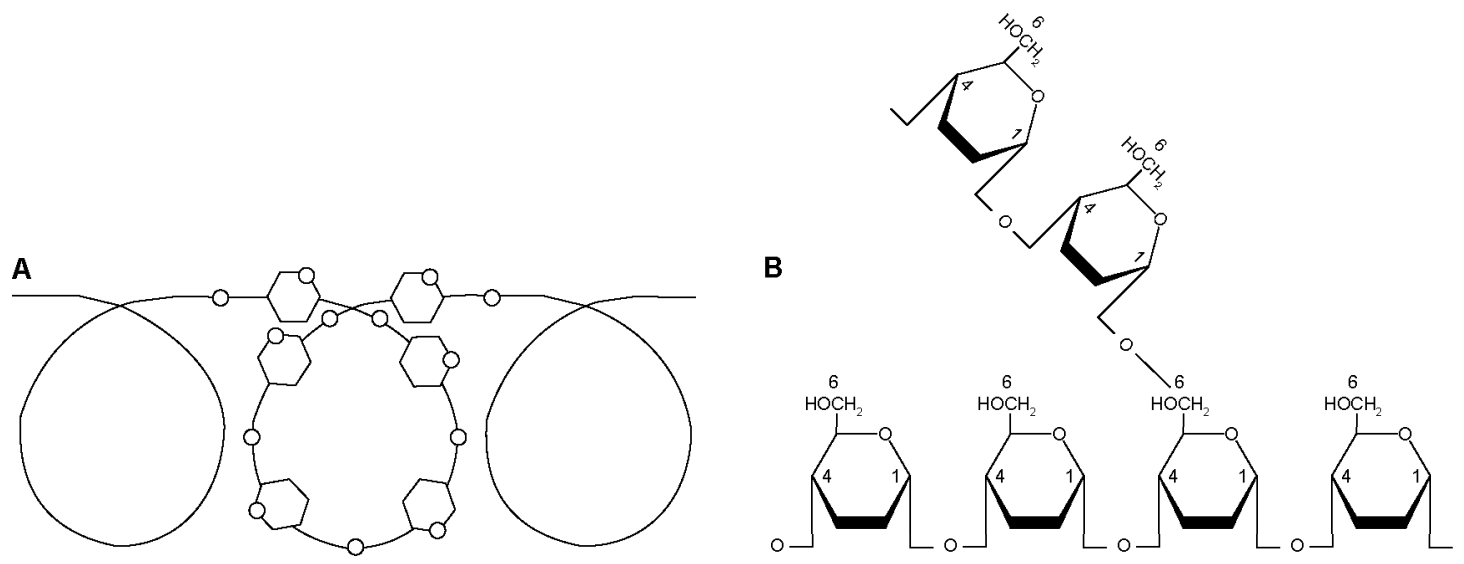

Figura 1. Estructura del almidón: a) estructura de la amilosa; b) estructura de la amilopectina.

Fuente: Murray, Bender y Botham (2000). 
Tabla 3. Propiedades de amilosa y amilopectina en la estructura del almidón

\begin{tabular}{lll}
\hline \multicolumn{1}{c}{ Propiedad } & \multicolumn{1}{c}{ Amilosa } & \multicolumn{1}{c}{ Amilopectina } \\
\hline Estructura molecular & Esencialmente lineal & Ramificada \\
Longitud promedio de cadena & 103 & $20-25$ \\
Grado de polimerización & 103 & $104-105$ \\
Estabilidad en solución acuosa & Retrogradación Fácilmente & Estable \\
Propiedades de película & Fuerte & Quebradiza \\
\hline
\end{tabular}

Fuente: Biliaderis (1991).

Las propiedades fisicoquímicas de cada almidón dependen en gran medida de su variedad y origen (BeMiller, 2016); estas propiedades son un factor diferencial que determina la funcionalidad o aplicabilidad de los almidones en las industrias.

En aplicaciones como la fabricación de estructuras biodegradables las propiedades físicas del almidón nativo juegan un papel importante (Willett, 2009); específicamente, algunas propiedades como el contenido de amilosa, el diámetro de partícula, hinchamiento de los granos, la temperatura de gelatinización, el pegado y la retrodegración (Biliaderis, 2009) hacen que el almidón sea funcional y tenga el potencial para ser una materia prima en este campo, sin la necesidad de realizar una modificación previa. En el caso de que las propiedades del almidón no sean aptas para diversas aplicaciones funcionales, se pueden realizar modificaciones físicas, químicas o enzimáticas (BeMiller, 2016; Chiu y Solarek, 2009) con el propósito de mejorar estas propiedades y especialmente su aplicabilidad.

\section{Modificación almidones}

El almidón es susceptible a cinco tipos de modificaciones: químicas, físicas, enzimáticas, genéticas o una combinación de estas (BeMiller, 2016; Kaur y Singh, 2016; Masina et ál., 2017). Las modificaciones químicas consisten en reacciones de oxidación, reticulación, estabilización y despolimerización, mientras que las modificaciones físicas involucran diferentes tipos de tratamientos térmicos (BeMiller, 2016).

Modificación química. Las modificaciones químicas son la técnica de modificación más usada debido a que no desnaturaliza el almidón e incrementa su funcionalidad (Masina et ál., 2017).

Se modifican los tres grupos hidroxilo disponibles en la posición $\mathrm{C}_{2}, \mathrm{C}_{3} \mathrm{y}_{6}$; el grado de modificación de estos grupos fluctúa debido al origen genético del almidón y las condiciones de reacción (Masina et ál., 2017).

Las reacciones de oxidación consisten en una disminución en la asociación de las moléculas de amilosa, que conduce a una estabilidad del almidón por medio de la adición de pequeñas cantidades funcionales que permiten conectar dos grupos hidroxilos de moleculas te Investigador y Dde grupos carbonilo y carboxilo dor y D(BeMiller, 2016).

La reticulación ocurre cuando los gránulos de almidón reaccionan con reactivos bifuncionales, que permiten conectar dos grupos hidroxilos de moléculas diferentes dentro del grano (BeMiller, 2016).

La estabilización del almidón es una reacción de sustitución; esta reacción confiere diferentes propiedades al almidón, ya que depende del tipo de almidón, $\mathrm{pH}$, concentración de reactivos, tiempo de la reacción. Un ejemplo de esta modificación es la esterificación del almidón con anhídrido acético en presencia de un catalizador alcalino (BeMiller, 2016; Kaur y Singh, 2016) .

La figura 2 presenta un resumen de las principales modificaciones químicas que puede tolerar el almidón, teniendo en cuenta las reacciones de esterificación, eterificación y oxidación de los grupos hidroxilos disponibles en la molécula del almidón. 


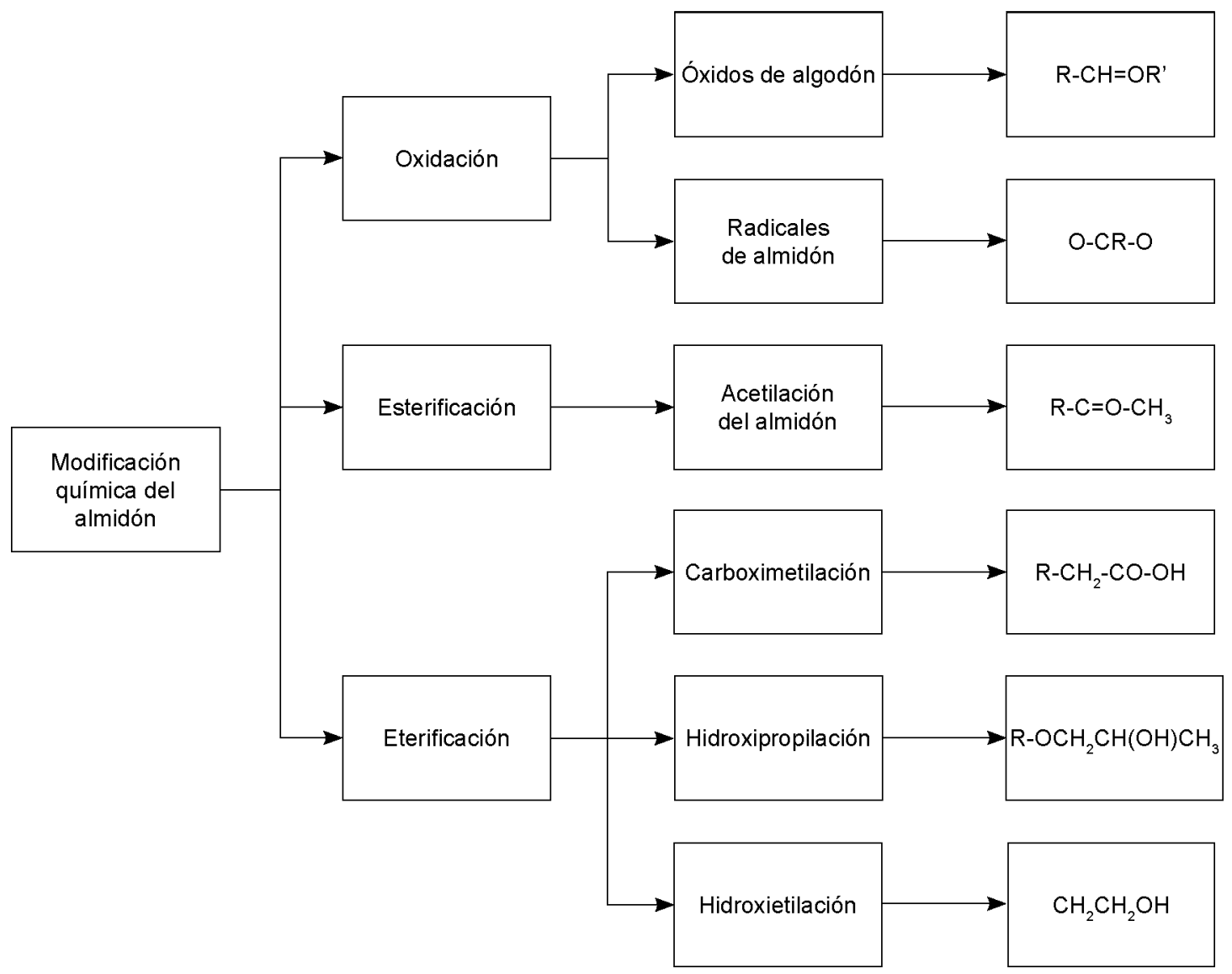

Figura 2. Modificaciones químicas comunes del almidón.

Fuente: Masina et ál. (2017).

Modificaciones fisicas. Las modificaciones físicas no involucran ninguna modificación en la estructura de la amilosa y la amilopectina; por tal motivo se implementan métodos térmicos para esta modificación (Kaur y Singh, 2016).

Se utilizan tratamientos hidrotérmicos y de presión como cocción por extrusión, sonicación y radiación. Cada procedimiento define las condiciones de agua, presión, temperatura y fuerza. También se utilizan tratamientos ANN (recocido) y HMT (tratamiento térmico de la humedad), los cuales modifican las propiedades fisicoquímicas del almidón sin destruir la estructura del grano (Kaur y Singh, 2016).

La dispersión en agua fría le proporciona mayor viscosidad y textura más lisa al almidón (Kaur y Singh, 2016).

\section{Almidón de quinua (Chenopodium quinua Willd.)}

La quinua, considerada un pseudocereal (Abugoch, 2009), es originaria de los Andes y ha sido cultivada desde hace 3000 a 4000 años (Li et ál., 2016). Chenopodium Quinua Willd. es identificada como un alimento potencial, debido al contenido en macro y micronutrientes y fitoconstituyentes, razón por la que es un alimento completo (Ahamed, Singhal, Kulkarni, Kale y Pal, 1996). La semilla de quinua está conformada por tres secciones: el embrión, endospermo y perisperma (Ando 
et ál., 2002; Prego, Maldonado y Otegui, 1998). En el perisperma, a diferencia de los cereales, se encuentra localizado el almidón y representa entre 53.5-69.2\% del peso seco de grano-semilla ( $\mathrm{Li}$ et ál., 2016); el gránulo de almidón mide 1-3 $\mu \mathrm{m}$ (Araujo-Farro, Podadera, Sobral y Menegalli, 2010) y 1.2-1.8 $\mu \mathrm{m}$ en el caso de las variedades colombianas (Bernal, Duarte, y Ramírez, 2015). También puede encontrase el almidón en aglomeraciones de entre $20-25 \mu \mathrm{m}$ ( $\mathrm{Li}$ et ál., 2016). El contenido de amilosa es 10-21 \% (Araujo-Farro et ál., 2010) o 4-25 \% (Li et ál., 2016), dependiendo de la variedad del grano de quinua.

E1 CBIQA ha definido un proceso de extracción de almidón nativo, escala de banco, partiendo de la evaluación del proceso de alistamiento de la matriz granos-semillas de quinua: limpieza y clasificación (Jiménez, Ochoa, Pava y Bernal, 2015), secado (Martínez, Ochoa, Trujillo y Bernal B., 2015), molienda de granos-semillas, mezclado y filtrado (González, Parrado y Morales, 2016) y secado crudo de almidón nativo (Borraez, Mejía, Ochoa y Bernal, 2016), con el propósito de desarrollar las tecnologías.

También se ha definido un patrón de uso a través de modificaciones físicas, químicas (Bernal, Cárdenas, Rozo, Daza y Echeverry, 2014; Duarte, Bernal y Ramirez, 2015; Pastás, Bernal y Pineda, 2012) y enzimáticas (Cuellar y Jaramillo, 2017), por las que este almidón puede ser utilizado en diferentes sectores industriales (Tafur y Bernal, 2017), por ejemplo: aditivos para alimentos (Acero y Guzman, 2015); obtención de octenilsuccinato alumínico de almidón de quinua, que es usado por la industria de cosméticos (Bernal et ál., 2014; Bernal, Leal, y Garzón, 2009; Daza, Bernal y Echeverri, 2012), y otros derivados que usa la industria de los hidrocarburos (Bernal, Ramírez, Duarte y Guzmán, 2015).

\section{DesarRollo del tema}

La revisión de las propiedades fisicoquímicas de los diferentes almidones determinó que el tamaño (diámetro) de gránulo oscila entre 1 y $110 \mu \mathrm{m}$. El almidón de papa tiene los diámetros más grandes, 20-110 $\mu \mathrm{m}$ (Singh et ál., 2003), y los más pequeños corresponden al almidón de quinua, 1-3 $\mu \mathrm{m}$ (Li et ál., 2016) y 1.2-1.8mm para variedades colombianas (Bernal et ál., 2015). Esta característica es fundamental en la elaboración de películas, debido a que un tamaño de grano más pequeño puede ser útil para una fácil dispersión y permite obtener mejores propiedades mecánicas (Ahamed et ál., 1996; Araujo-Farro et ál., 2010; Medina y Salas, 2008). Además de lo indicado, el tamaño de granulo interviene en la solubilidad y el poder de hinchamiento, lo que afecta la fase amorfa de la estructura (Medina y Salas, 2008).

El contenido de amilosa para el grupo de almidones que no han sido objeto de modificaciones oscila entre 7.8 y $32.5 \%$, valores que corresponden al almidón de amaranto (Qian y Kuhn, 1999) y al almidón de maíz (Singh et ál., 2003), respectivamente. Esta macromolécula afecta las propiedades mecánicas y el porcentaje de transparencia en las películas a base de almidón (Mali, Karam, Ramos y Grossmann, 2004); así, a medida de que el contenido de amilosa aumenta, la transparencia de la película disminuye (Swinkels, 1985), mientras que al aumentar el contenido de amilosa, las propiedades mecánicas (mayor módulo, resistencia a la tracción y resistencia al impacto) y térmicas de la película mejoran (Wittaya,2012) debido a la iteración de la amilosa lineal con los puentes de hidrogeno; por consiguiente, las películas no plastificadas ricas en amilosa son más rígidas y fuertes (Lourdin et ál., 1995; Rindlav-Westling, Stading, Hermansson y Gatenholm, 1998).

La tabla 2 expone diferentes formulaciones que se pueden tener en cuenta en la elaboración de películas a partir de almidón, en las que el agente plastificante, las condiciones de gelatinización y las condiciones de secado tienen un papel fundamental en las condiciones del proceso (Jiménez et ál., 2012). La gelatinización de un almidón se puede definir como el proceso con el que un almidón pierde su cristalinidad; igualmente, la absorción de gran cantidad de agua provoca una hinchazón 
en sus granos y un aumento de volumen (Hernández-Medina et ál., 2008). En esta operación se libera amilosa y amilopectina. La amilopectina ramificada no interactúa, por lo que forma un gel que es débil y flexible; por el contrario, las cadenas lineales de amilosa interactúan y forman geles más rígidos y fuertes con respecto a los de amilopectina (Mali et ál., 2004; Rindlav-Westling et ál., 1998). Este procedimiento requiere una carga calórica suministrada por un aumento de temperatura que varía según el tipo de almidón (Hernández-Medina et ál., 2008). Para los almidones evaluados a la temperatura pico de gelatinización el rango varía de 84.25 y $59.2^{\circ} \mathrm{C}$, en el que la mayor temperatura corresponde a los almidones que tiene diámetros de partícula grandes, como el plátano (Tanduk) $\left(84.25^{\circ} \mathrm{C}\right.$ ) (Shittu et ál., 2016), y la menor, a los diámetros de partículas pequeños, como el almidón de quinua $\left(59.2^{\circ} \mathrm{C}\right.$ ) (Li et ál., 2016).

\section{Resultados}

A partir de la revisión de las principales propiedades involucradas en la elaboración de estructuras poliméricas tipo película, se puede postular al almidón de quinua como una materia prima potencial para este proceso debido a que tiene propiedades físicas diferenciales a comparación de los demás almidones analizados. Para el desarrollo de estas estructuras, también es necesario considerar diferentes variables que dependen de la utilidad final que se le quiera dar a la estructura. Las principales variables a tener en cuenta son: contenido de almidón, uso de plastificantes, cantidad del plastificante, tiempo de secado, pH, entre otras (Araujo-Farro et ál., 2010; Meneses et ál., 2007).

\section{Conclusiones}

Los almidones son una reserva energética de plantas que puede ser modificada por vía física, química o enzimática, para mejorar sus propiedades y poderla implementar en diferentes campos de acción (alimentos, cosmética, petróleo, producción de materiales biodegradables).

Como resultado de esta investigación, se puede concluir que la diferencia en las propiedades fisicoquímicas de los almidones permite obtener estructuras poliméricas (sean termoplásticos o películas) funcionales en diversos campos de aplicación.

Para la fabricación de estructuras poliméricas tipo películas se deben considerar tres propiedades fundamentales: contenido de amilosa, diámetro de partícula y temperatura de gelatinización, ya que estas determinan sustancialmente el comportamiento mecánico y óptico del material elaborado.

Se determinó que el almidón de quinua puede ser una materia prima para la elaboración de estructuras poliméricas tipo películas con potencial biodegradable, debido a que la materia prima base es un polímero de origen natural. Sumado a esto, este almidón tiene diámetros de partícula pequeños $(1-3 \mu \mathrm{m})$, su temperatura de gelatinización es baja y, al tener un valor intermedio de amilosa (10-21.2\%), se puede obtener películas semitransparentes y con una moderada resistencia mecánica.

\section{Agradecimientos}

Los autores, miembros del CBIQA (línea de investigación en Estructuras Híbridas Poliméricas), agradecen la colaboración del Sistema de Biblioteca Baldomero Sanín Cano, de la Fundación Universidad de América, por su colaboración en la búsqueda de información y por los procesos didácticos y pedagógicos seguidos durante el entrenamiento en bibliometría y consulta de la información. 


\section{REFERENCIAS}

Abugoch, L. (2009). Quinoa (Chenopodium quinoa Willd.): Composition, chemistry, nutritional, and functional properties. En S. Taylor (ed.), Advances in Food and Nutrition Research (1 ${ }^{\mathrm{a}} \mathrm{ed}$. pp. 1-31). EE. UU.: Elsevier Inc.

Acero, J., y Guzmán, S. (2015). Hidrolisis enzimatica de almidón nativo de quinua, para obtener un producto con propiedades reologicas de una grasa alimentaria (tesis de pregrado). Fundación Universidad de América, Bogotá, Colombia.

Ahamed, N., Singhal, R., Kulkarni, P., Kale, D., y Pal, M. (1996). Studies on Chenopodium quinoa and Amaranthus paniculatas starch as biodegradable fillers in LDPE films. Carbohydrate Polymers, 31(3), 157-160. https://doi.org/10.1016/S0144-8617(96)00019-7

Alvis, A., Vélez, C., Villada, H., y Rada-Mendoza, M. (2008). Análisis físico-químico y morfológico de almidones de ñame, yuca y papa y determinación de la viscosidad de las pastas. Informacion Tecnologica, 19(1), 19-28. https://doi.org/10.4067/S0718-07642008000100004

Ando, H., Chen, Y.-C., Tang, H., Shimizu, M., Watanabe, K., y Mitsunaga, T. (2002). Food Components in Fractions of Quinoa Seed. Food Science and Technology Research, 8(1), 80-84. https://doi.org/10.3136/fstr.8.80

Araujo-Farro, P., Podadera, G., Sobral, P., y Menegalli, F. (2010). Development of films based on quinoa (Chenopodium quinoa, Willdenow) starch. Carbobydrate Polymers, 81(4), 839-848. https://doi.org/10.1016/j.carbpol.2010.03.051

Averous, L. , Fauconnier, N., Moro, L., y Fringant, C. (2000). Blends of thermoplastic starch and polyesteramide: processing and properties. Journal of Applied Polymer Science, 76(7), 1117-1128. https://doi.org/10.1002/(SICI)1097-4628(20000516)76:7<1117::AIDAPP16>3.0.CO;2-W

Basiak, E., Lenart, A., y Debeaufort, F. (2017). Effect of starch type on the physico-chemical properties of edible films. International Journal of Biological Macromolecules, 98, 348-356. https:// doi.org/10.1016/j.ijbiomac.2017.01.122

BeMiller, J. (2016). Starch, Modification. Reference Module in Food Science (2a ed.). https://doi. org/10.1016/B978-0-08-100596-5.00147-5

Bernal, C., Leal, A., y Garzón, J. (2009). Obtención, a escala de laboratorio, de octenilsuccinato alumínico de almidón de quinua, con miras a su utilización en un producto cosmético. Revista Virtual Pro, 95, 15-34.

Bernal, C., Cárdenas, O., Rozo, W., Daza, P., y Echeverry, A. (2014). Octenilsuccinato alumínico de almidón de quinua -grado cosmético- y su certificación bajo la denominación de ingrediente naturál. Revista de Investigación, 7(2), 38-49.

Bernal, C., Duarte, D., Ramírez, L., Guzmán, A. (2015). Quinua, Chenopodium quinua (Willd.) en Colombia. Caracterizacion de gránulos de almidón nativo de quinua por IR-ATR, MEB, DRX. Revista de Investigación, 8(2), 122-131.

Bernal, C., Villegas, E., Sandoval, G., Lache, A. y Correa, C. (2015). Quinua (Chenopodium quinoa Willd) en Colombia. Primera Entrega: un nuevo enfoque de negocio. Revista Virtual Pro, $164,1-31$. 
Bernal, C., Ramírez, L., Duarte, P., y Guzmán, A. (2015). Quinua, Chenopodium quinua (Willd.) en Colombia: caracterización de granulos de almidón nativo de quinua por IR-ATR, MEB,DRX. Revista de Investigación, 8(2), 122-131.

Biliaderis, C.. (1991). The structure and interactions of starch with food constituents. Canadian Journal of Physiology and Pharmacology, 69(1), 60-78. https://doi.org/10.1139/y91-011

Biliaderis, C. (2009). Structural transitions and related physical properties of starch. En J. BeMiller y R. Whistler (eds.), Food Science and Technology (3 $3^{a}$ edición, pp. 293-372). San Diego, EE. UU.: Academic Press.

Bootklad, M., Chantarak, S., y Kaewtatip, K. (2016). Novel biocomposites based on wheat gluten and rubber wood sawdust. Journal of Applied Polymer Science, 133(30), 1-6. https://doi. org/10.1002/app.43705

Borraez, C., Mejía, N., Ochoa, J., y Bernal, C. (2016). Desarrollo de una tecnología escala de banco para el secado de crudo de almidón nativo de quinua (tesis de pregrado). Fundación Universidad de América, Bogotá D. C., Colombia.

Buléon, A., Colonna, P., Planchot, V., y Ball, S. (1998). Starch granules: Structure and biosynthesis. International Journal of Biological Macromolecules, 23(2), 85-112. https://doi.org/10.1016/ S0141-8130(98)00040-3

Chávez-Salazar, A., Bello-Pérez, L., Agama-Acevedo, E., Castellanos-Galeano, F., Álvarez-Barreto, C., y Pacheco-Vargas, G. (2017). Isolation and partial characterization of starch from banana cultivars grown in Colombia. International Journal of Biological Macromolecules, 98 , 240-246.

Chiu, C., y Solarek, D. (2009). Modification of Starches BT - Starch. En J. BeMiller y R. Whistler (eds.), Food Science and Technology (3a edición, pp. 629-655). San Diego, EE. UU.: Academic Press.

Cuellar, L., y Jaramillo, S. (2017). Establecimiento del modelo cinetico de la bidrolisis parcial enzimatica del almidon nativo de quinua, funcionalizado a grado alimentario (tesis de pregrado). Fundación Universidad de América, Bogota, D. C., Colombia.

Daza, P., Berna, C., y Echeverri, A. (2012). Evaluación del proceso de obtención de octenilsuccinato alumínico de almidón de Quinua-Osa Quinua-, grado cosmético, para la obtención del producto apto para su certificación (tesis de pregrado). Fundación Universidad de América, Bogotá, D. C., Colombia.

Duarte, P., Bernal, C., y Ramírez, L. (2015). Obtención de carboximetil almidón de quinua, a escala laboratotio, a partir de almidón nativo de Chenopodium quinoa (Willd.) para su uso en la industria de hidrocarburos comoa ditivo en fuidos de perforación (tesis de pregrado). Fundación Universidad de América, Bogotá D. C., Colombia.

Fakhouri, F., Martelli, S., Caon, T., Velasco, J., y Mei, L. (2015). Edible films and coatings based on starch/gelatin: Film properties and effect of coatings on quality of refrigerated Red Crimson grapes. Postharvest Biology and Technology, 109, 57-64. https://doi.org/10.1016/j.postharvbio.2015.05.015

Franklin, M., Pushpadass, H., Kumar, B., Kulkarni, S., Muthurayappa, M., Kandasamy, R., Venkatachalam, R., y Vellingiri, P. (2017). Physicochemical, thermal, pasting and microstructural 
characterization of commercial Curcuma angustifolia starch. Food Hydrocolloids, 67, 27-36. https://doi.org/10.1016/j.foodhyd.2016.12.025

Pacheco, G., Flores, N.., y Romina, R. (2014). Bioplásticos. BioTecnologia, 18(2), 27-36.

González, A., Parrado, J. y Morales, M. (2016). Desarrollo tecnologico a escala laboratorio de un sistema de mezclado y filtrado de crudo de almidón proveniente de granos semilla de la especie Chenopadium Quinua (Wild) (tesis de pregrado). Fundación Universidad de América, Bogotá D. C., Colombia

González-Gutierrez, J., Partal, P., Garcia-Morales, M., y Gallegos, C. (2010). Development of highly-transparent protein/starch-based bioplastics. Bioresource Technology, 101(6), 20072013. https://doi.org/10.1016/j.biortech.2009.10.025

Hernández-Medina, M., Torruco-Uco, J. G., Chel-Guerrero, L., y Betancur-Ancona, D. (2008). Caracterización fisicoquímica de almidones de tubérculos cultivados en Yucatán, México. Ciência e Tecnologia de Alimentos, 28(3), 718-726. https://doi.org/10.1590/S010120612008000300031

Hoornweg, D., y Bhada, P. (2012). What a Waste. A global review of solid waste management (Urban Development Series Knowledge Papers) .https://doi.org/10.1111/febs.13058

Hoover, R. (2001). Composition, molecular structure, and physicochemical properties of tuber and root starches: A review. Carbohydrate Polymers, 45(3), 253-267. https://doi.org/10.1016/ S0144-8617(00)00260-5

Jane, J. (2009). Structural features of starch granules II. Starch (3a edición). Recuperado de https:// doi.org/10.1016/B978-0-12-746275-2.00006-9

Jiménez, J., Ochoa, J., Pava, S., y Bernal, C. (2015). Diseño de una máquina clasificadora, por dimensiones, de la semilla de quinua usando una energía alternativa (tesis de pregrado). Fundación Universidad de América, Bogotá D. C., Colombia.

Jiménez, A., Fabra, M., Talens, P., y Chiralt, A. (2012). Edible and biodegradable starch films: A review. Food and Bioprocess Technology, 5(6), 2058-2076. https://doi.org/10.1007/s11947012-0835-4

Kaur, L., y Singh, J. (2016). Starch: Modified Starches. Encyclopedia of Food and Health (1 ${ }^{a}$ edición). Recuperado de https://doi.org/10.1016/B978-0-12-384947-2.00659-0

Li, G., Wang, S., y Zhu, F. (2016). Physicochemical properties of quinoa starch. Carbohydrate Polymers, 137,328-338. https://doi.org/10.1016/j.carbpol.2015.10.064

Lourdin, D., Valle, G. Della, y Colonna, P. (1995). Influence of amylose content on starch films and foams. Carbohydrate Polymers, 27(4), 261-270. https://doi.org/10.1016/0144$8617(95) 00071-2$

Mali, S., Karam, L, Ramos, L., y Grossmann, M. (2004). Relationships among the composition and physicochemical properties of starches with the characteristics of their films. Journal of Agricultural and Food Chemistry, 52(25), 7720-7725. https://doi.org/10.1021/jf049225+

Martínez, N., Ochoa, J., Trujillo, A., y Bernal, C. (2015). Diseño de una máquina para el secado de la semilla de quinua usando una energía alternativa (tesis de pregrado). Programa de Ingeniería Mecánica, Fundación Universidad de América, Bogotá, D. C., Colombia. 
Martins, L., Rodrigues, V., Jardim, A., Mossmann, A., Aparecida, L., Henkes, A., Cordeiro Luiz, C., Ferreira A., da Rosa, E. (2016). Mechanical Analysis of Biodegradable Films from Native and Chemically Modified Potato Starches. Materials Sicience Forum, 869, 830-834. https://doi.org/10.4028/www.scientific.net/MSF.869.830

Masina, N., Choonara, Y.., Kumar, P., du Toit, L., Govender, M., Indermun, S., y Pillay, V. (2017). A review of the chemical modification techniques of starch. Carbohydrate Polymers, 157, 1226-1236. https://doi.org/10.1016/j.carbpol.2016.09.094

Mathew,A., y Dufresne, A. (2002). Plasticized waxy maize starch: Effect of polyols and relative humidity on material properties. Biomacromolecules, 3(5), 1101-1108. https://doi.org/10.1021/ bm020065p

Medina, J., y Salas, J. (2008). Caracterización morfológica del granulo de almidón nativo: apariencia, forma, tamaño y su distribución. Revista de Ingeniería, 27, 56-62.

Meneses, J., Corrales, C., y Valencia, M. (2007). Síntesis y caracterización de un polímero biodegradable a partir del almidón de yuca. Revista EIA, 8, 57-67.

Murray, R., Bender, D., Botham, K., Kennelly, P., Rodwell, V., y Weil, A. (2000). Harper: bioquimica ilustrada (29a edición). [s. d.]: McGraw-Hill Interamericana.

Comisión Europera. (2015). Greener polymers (Reporte final de NanoBioComp, ID 273715). Recuperado de http://cordis.europa.eu/result/rcn/151411_en.html

Nwokocha, L. M., y Williams, P. A. (2009). Some properties of white and yellow plantain (Musa paradisiaca, Normalis) starches. Carbobydrate Polymers, 76(1), 133-138. https://doi. org/10.1016/j.carbpol.2008.10.012

Palma-Rodríguez, H., Aguirre-Álvarez, G., Chavarría-Hernández, N., Rodríguez-Hernández, A., Bello-Pérez, L., y Vargas-Torres, A. (2012). Oxidized banana starch-polyvinyl alcohol film: Partial characterization. Starch/Staerke, 64(11), 882-889. https://doi.org/10.1002/ star.201200035

Pastás, N., Bernal, C., y Pineda, Y. (2012). Desarrollo de un polvo cosmético compacto empleando octenilsuccinato aluminico de almidón de quinua (tesis de pregrado). Programa de Ingeniería Química, Fundación Universidad de América, Bogotá, D. C., Colombia.

Patni, N., Yadava, P., Agarwal, A., y Maroo, V. (2014). An overview on the role of wheat gluten as a viable substitute for biodegradable plastics. Reviews in Chemical Engineering, 30(4), 421430. https://doi.org/10.1515/revce-2013-0039

Pelissari, F., Andrade-Mahecha, M., Amaral, P., y Menegalli, F. (2013). Comparative study on the properties of flour and starch films of plantain bananas (Musa paradisiaca). Food Hydrocolloids, 30(2), 681-690. https://doi.org/10.1016/J.FOODHYD.2012.08.007

Penaranda, O., Perilla, J., y Algecira, N. (2008). A review of using organic acids to chemically modify starch. Ingenieria e Investigación, 28(3), 47-52.

Podshivalov, A., Zakharova, M., Glazacheva, E., y Uspenskaya, M. (2017). Gelatin/potato starch edible biocomposite films: correlation between morphology and physical properties. Carbohydrate Polymers, 157, 1162-1172. https://doi.org/10.1016/j.carbpol.2016.10.079

Prego, I., Maldonado, S., y Otegui,M. (1998). Seed Structure and Localization of Reserves in Chenopodium quinoa. Annals of Botany, 82, 481-488. https://doi.org/10.1006/anbo.1998.0704 
Qian, J., y Kuhn, M. (1999). Characterization of Amaranthus cruentus and Chenopodium quinoa Starch. Starch-Stärke, 51(4), 116-120. https://doi.org/10.1002/(SICI)1521379X(199904)51:4<116::AID-STAR116>3.0.CO;2-R

Ramírez-Hernández, A., Aparicio-Saguilán, A., Reynoso-Meza, G., y Carrillo-Ahumada, J. (2017). Multi-objective optimization of process conditions in the manufacturing of banana (Musa paradisiaca L.) starch/natural rubber films. Carbohydrate Polymers, 157, 1125-1133. https://doi.org/10.1016/J.CARBPOL.2016.10.083

Rindlav-Westling, A., Stading, M., Hermansson, A.-M., y Gatenholm, P. (1998). Structure, mechanical and barrier properties of amylose and amylopectin films. Carbohydrate Polymers, 36(2), 217-224. https://doi.org/https://doi.org/10.1016/S0144-8617(98)00025-3

Robyt, J. (2009). Enzymes and Their Action on Starch. En J. BeMIller y R. Whistler (eds.), Starch, (3a edición, pp. 237-292). EE. UU.: Academic Press.

Romero-Bastida, C., Zamudio-Flores, P., y Bello-Pérez, L. (2011). Antimicrobials in oxidized banana starch films: effect on antibacterial activity, microstructure, mechanical and barrier properties. Revista Mexicana de Ingeniera Química, 10(3), 445-453.

Rubio, M. y, y Guerrero, J. A. (2012). Polímeros utilizados para la elaboración de películas biodegradables. Temas Selectos de Ingenieria de Alimentos, 2, 173-181.

Sessini, V., Arrieta, M. P., Kenny, J. M., y Peponi, L. (2016). Processing of edible films based on nanoreinforced gelatinized starch. Polymer Degradation and Stability, 132(February), 157168. https://doi.org/10.1016/j.polymdegradstab.2016.02.026

Shittu, R., Lasekan, O., Karim, R., y Sulaiman, R. (2016). Plantain-starch: Microstructural, physicochemical, and morphological characteristics of two cultivars grown in Malaysia. StarchStärke, 68(11-12), 1187-1195. https://doi.org/10.1002/star.201500262

Singh, N., Singh, J., Kaur, L., Sodhi, N. S., y Gill, B. (2003). Morphological, thermal and rheological properties of starches from different botanical sources. Food Chemistry, 81(2), 219-231. https://doi.org/10.1016/S0308-8146(02)00416-8

Stagner, J., Alves, V., y Narayan, R. (2012). Application and performance of maleated thermoplastic starch-poly (butylene adipate- co-terephthalate) blends for films. Journal of Applied Polymer Science, 126(S1), E135-E142. https://doi.org/10.1002/app.34876

Swinkels, J. (1985). Composition and properties of commercial native starches. Starch-Stärke, 37(1), 1-5. https://doi.org/10.1002/star.19850370102

Tafur, L., y Bernal, C. (2017). Ajuste de un modelo matemático al fenomeno de extraccion sólido-líquido del almidon nativo de quinua, base aua, a partir de granos-semillas de la especie Chenopodium quinoa (willd), por medio de un diseño experimentál (tesis de pregrado). Programa de Ingeniería Química, Fundación Universidad de América, Bogota, D. C., Colombia.

Tan, I., Torley, P., y Halley, P. (2008). Combined rheological and optical investigation of maize, barley and wheat starch gelatinisation. Carbohydrate Polymers, 72(2), 272-286. https://doi. org/10.1016/j.carbpol.2007.08.018

Willett, J. (2009). Starch in Polymer Compositions1 BT-Starch. EnFood science and technology (3a edición, pp. 715-743). San Diego, EE. UU.: Academic Press. https://doi.org/https://doi. org/10.1016/B978-0-12-746275-2.00019-7 
Wittaya, T. (2012). Rice Starch-Based Biodegradable Films: Properties Enhancement. En A. Amer (ed.), Structure and function of food engineering (pp. 103-134). https://doi.org/10.5772/47751. hancement 